\section{Utility of the AVISE Connective Tissue Disease test in predicting lupus diagnosis and progression}

\author{
Emily Liang, ${ }^{1}$ Mihaela Taylor, ${ }^{2}$ Maureen McMahon (i) ${ }^{2}$
}

To cite: Liang E, Taylor M, McMahon M. Utility of the AVISE Connective Tissue Disease test in predicting lupus diagnosis and progression. Lupus Science \& Medicine 2020;7:e000345. doi:10.1136/ lupus-2019-000345

- Additional material is published online only. To view, please visit the journal online (http://dx.doi.org/10.1136/ lupus-2019-000345).

Received 14 June 2019 Revised 3 December 2019 Accepted 3 December 2019
Check for updates

(C) Author(s) (or their employer(s)) 2020. Re-use permitted under CC BY-NC. No commercial re-use. See rights and permissions. Published by BMJ.

${ }^{1}$ David Geffen School of Medicine at UCLA, Los Angeles, California, USA

${ }^{2}$ Internal Medicine, Division Rheumatology, University of California, Los Angeles, Los Angeles, California, USA

Correspondence to Dr Maureen McMahon; mmcmahon@mednet.ucla.edu

\section{ABSTRACT}

Background The AVISE Connective Tissue Disease (CTD) test uses autoantibody, erythrocyte-bound C4d (EC4d) and B-cell-bound C4d (BC4d) levels to aid in diagnoses of SLE, other CTDs and fibromyalgia. We evaluated the utility of the AVISE CTD test in predicting SLE disease development and damage progression.

Methods Patients who had undergone AVISE CTD testing were assessed for SLE diagnosis by the Systemic Lupus International Collaborating Clinics (SLICC) and American College of Rheumatology criteria and for SLE damage by the Systemic Lupus International Collaborating Clinics Damage Index (SDI) at the time of AVISE testing $(t=0)$ and 2 years later $(\mathrm{t}=2)$.

Results Among 117 patients without a previous diagnosis of SLE, $65 \%$ of patients who tested positive developed SLE at $\mathrm{t}=2$, compared with $10.3 \%$ of patients who tested non-positive $(p<0.0001)$. AVISE-positive patients fulfilled significantly more SLICC diagnostic criteria than AVISEnon-positive patients at both $\mathrm{t}=0$ (3.8 2.1 vs $1.9 \pm 1.1$, $p=0.001)$ and $t=2(4.5 \pm 2.2$ vs $2.1 \pm 1.2, p<0.0001)$. AVISEpositive patients also had had significantly higher SDI at $t=2(1.9 \pm 1.3$ vs $1.03 \pm 1.3, p=0.01)$. BC4d levels correlated with the number of SLICC criteria at $\mathrm{t}=0(\mathrm{r}=0.33$, $p<0.0001)$ and $t=2(r=0.34, p<0.0001)$, as well as SDI at $\mathrm{t}=0(\mathrm{r}=0.25, \mathrm{p}=0.003)$ and $\mathrm{t}=2(\mathrm{r}=0.26, \mathrm{p}=0.002)$.

Conclusions The AVISE CTD test can aid in SLE evaluation by predicting SLE disease development and future damage progression.

\section{INTRODUCTION}

SLE is an autoimmune condition that can damage multiple organ systems. ${ }^{1}$ Classification criteria, including clinical and immunological criteria, have been developed by SLICG and American College of Rheumatology (ACR) to help identify patients with SLE. ${ }^{2}$ Despite these criteria, initial diagnosis of SLE can be challenging due to symptoms that are non-specific, do not meet classification criteria or overlap with other connective tissue diseases (CTDs). In fact, up to $50 \%$ of patients with CTDs have an unclassifiable profile at disease onset. ${ }^{3}$ Because early diagnosis of SLE decreases rates of flares, hospitalisations and healthcare costs, ${ }^{4}$ the development of new diagnostic tests for SLE has become a key area of interest.

Scoring systems have also been developed to assess disease activity in SLE based on the presence of certain clinical features attributable to SLE. The Systemic Lupus Activity Measure, European Community Lupus Activity Measure and Systemic Lupus Erythematosus Disease Activity Index 2000 (SLEDAI-2K) calculate global indices, while the British Isles Lupus Assessment Group, which separates activity by organ systems, is based on the physician's intention to treat. ${ }^{56}$ Organ damage in SLE, defined as irreversible damage present for at least 6 months, is evaluated by the SDI. ${ }^{7}$

In addition to these scoring systems, laboratory tests are used to monitor disease activity and damage in SLE. Traditional measures such as low complement C3 and C4 levels, which reflect activation of the complement cascade due to ongoing inflammation, have been incorporated into the SLICC classification criteria and SLEDAI-2K. Antidouble-stranded DNA (dsDNA) antibodies have also been shown to precede flares in disease activity, particularly in lupus nephritis. ${ }^{8}$ However, drawbacks of these laboratory measures include low sensitivity and inability to predict flares in patients with serologically active clinically quiescent SLE. ${ }^{9}$ Hypocomplementemia as a measure of disease activity is also confounded by an increased synthesis during acute inflammation, genetic variation in baseline complement levels and activation by anticomplement autoantibodies rather than disease. ${ }^{1011}$

Recently, investigators have begun to explore the use of cell-bound complement activation products (CB-CAPs) as biomarkers of lupus diagnosis and activity. High levels of EC4d, B-cell-bound C4d (BC4d) and plateletbound C4d (PC4d) can differentiate SLE from other CTDs with 56\%-72\% sensitivity and $80 \%-98 \%$ specificity, and from healthy 
individuals with $60 \%-81 \%$ sensitivity and $91 \%-100 \%$ specificity. ${ }^{12}$ The combination of EC4d and BC4d has been shown to have higher sensitivity than C3/C4 and antidsDNA for SLE. ${ }^{10}$ CB-CAPs have also demonstrated high sensitivity and specificity in diagnosing childhood-onset SLE. ${ }^{13}$ Furthermore, longitudinal studies have shown that higher levels of EC4d, erythrocyte-bound C3d (EC3d) and reticulocyte-bound $\mathrm{C} 4 \mathrm{~d}$ are associated with greater disease activity, demonstrating the potential utility of CB-CAP biomarkers in monitoring disease activity. ${ }^{12} 14$ Lastly, PC4d levels have been demonstrated to correlate with history of seizure, positive antiphospholipid antibody tests and frequency of cardiovascular events. ${ }^{12}$

Developed by Exagen Diagnostics, the AVISE CTD test is a newly approved, commercially available test that combines autoantibody, EC4d, and BC4d levels to aid in challenging diagnoses of SLE, other CTDs and fibromyalgia (FM). It contains SLE-associated markers and extractable nuclear antigen, rheumatoid arthritis (RA), antiphospholipid syndrome and thyroid panels. This two-tiered test has been shown to have high sensitivity and specificity for differentiating SLE from patients with other CTDs or FM. ${ }^{15}$ However, there have been no studies assessing the clinical utility of the test in predicting future clinical diagnoses and outcomes in a real-world cohort. Therefore, in this study of a university-based cohort, we evaluated the utility of the AVISE CTD test in predicting SLE disease diagnosis and damage progression.

\section{METHODS}

This retrospective longitudinal observational study examined all patients from a single rheumatological provider who did not have a confirmed SLE diagnosis and who had undergone AVISE CTD testing as part of their routine clinical care between April 2014 and November 2016. The AVISE test was ordered for all patients within this time frame who had inflammatory arthritis, undifferentiated connective tissue disease (UCTD) or other diagnoses with symptoms or features that suggested a possible SLE diagnosis. AVISE testing was also performed in some patients with established lupus in order to monitor disease activity; these patients were included only in the analyses of damage progression (notations are included in the text/tables when included). For purposes of this analysis, a 'positive' AVISE result for SLE was identified as a positive or moderate positive score (tier 1 positive or tier 2 index $>0.1$ ). 'Non-positive' results were defined as negative, moderate negative, equivocal or indeterminate scores (tier 1 negative and tier 2 index $\leq 0.1$ ). EC4d, BC4d, dsDNA indirect fluorescent antibody (IFA) status (crithidia) and antibody titres (ELISA) and CCP antibody titres (ELISA) were collected as part of the AVISE testing panel.

Data were collected via a review of the patients' medical charts. The working diagnoses as noted in the medical record by the patients' treating physician were recorded at $\mathrm{t}=0$ and $\mathrm{t}=2$, where $\mathrm{t}=0$ reflected the suspected referral diagnosis at the time of the AVISE testing and $\mathrm{t}=2$ reflected the final diagnosis 2 years later. SLE diagnoses were confirmed using the SLICC/ACR classification criteria at $\mathrm{t}=0$ and $\mathrm{t}=2{ }^{2}$ Damage for all patients (regardless of confirmed SLE diagnosis) was evaluated using SDI at $\mathrm{t}=0$ and $\mathrm{t}=2$. Rheumatological medications at $\mathrm{t}=0$ and $\mathrm{t}=2$ were recorded as hydroxychloroquine, methotrexate, mycophenolate, other disease-modifying agents (diseasemodifying antirheumatic drugs), belimumab and RA biologics (such as adalimumab and tocilizumab). C3 and $\mathrm{C} 4$ levels at $\mathrm{t}=0$ were measured in the UCLA clinical laboratory.

\section{Statistical analysis}

Data were analysed using SPSS V.25.0. Skewed continuous variables were logarithmically transformed to attain a normal distribution (note: non-transformed data are presented in figures and tables to facilitate interpretation of results). For variables that did not attain a normal distribution by logarithmic transformation, non-parametric tests were used. Study groups were compared using analysis of Student's t-test for continuous parametric variables, Mann-Whitney test for non-parametric variables and the $\chi^{2}$ test or Fisher's exact test for categorical variables. The significance level was set at $\mathrm{p}<0.05$. Spearman's rank correlation was used to evaluate for correlations between biomarkers, SLICC criteria fulfilment and SDI.

\section{RESULTS}

\section{Study cohort characteristics}

The study cohort consisted of a total of 138 patients (130 women and eight men). Twenty-one patients had an established diagnosis of SLE at baseline; these patients were excluded from subsequent analyses. In the remaining 117 patients, the average age was 52.5 years, with an SD of 12.2 years (table 1 ). The most common diagnoses at $\mathrm{t}=0$ were UCTD (40.2\%), RA (21.4\%) and other autoimmune $(21.4 \%)$ (table 1). The most common medication at $\mathrm{t}=0$ was hydroxychloroquine $(51.2 \%)$, followed by an RA biologic $(14.5 \%)$, methotrexate $(8.5 \%)$ and belimumab $(5.8 \%)$ (table 1). Forty-four patients had a change in diagnosis at $\mathrm{t}=2$ (table 1$)$.

In those who had a positive AVISE test at baseline, the most common presumed diagnoses at $\mathrm{t}=0$ were UCTD, followed by other autoimmune diagnoses (palindromic rheumatism (1), polymyalgia rheumatica (1) and RA) (figure 1A). In the remaining patients who tested nonpositive, the most common diagnoses at $\mathrm{t}=0$ were UCTD, followed by RA and other autoimmune (including Sjogren's (7), inflammatory myopathy (1), palindromic rheumatism (2), seronegative spondyloarthritis (6), polymyalgia rheumatica (2), Still's (1), vasculitis (2) and scleroderma (2)) (figure 1B).

\section{Patients with a positive AVISE are more likely to have a} diagnosis of SLE at 2 years

In patients who did not have an established lupus diagnosis at baseline, the diagnoses changed from $t=0$ to $t=2$ 
Table 1 Characteristics of patients without a confirmed SLE diagnosis at baseline

\begin{tabular}{|c|c|c|c|}
\hline & $\begin{array}{l}\text { Positive AVISE } \\
(n=20)\end{array}$ & $\begin{array}{l}\text { Non-positive AVISE } \\
(\mathrm{n}=97)\end{array}$ & $P$ value \\
\hline \multicolumn{4}{|l|}{ Demographics } \\
\hline Average age at baseline & $52.7 \pm 12.1$ & $53.9 \pm 14.7$ & ns \\
\hline \multicolumn{4}{|l|}{ Baseline diagnoses at $\mathrm{t}=0 \%(\mathrm{n})$} \\
\hline UCTD & $65.0(13)$ & $35.1(34)$ & \\
\hline Other autoimmune & $10.0(2)$ & $23.7(23)$ & \\
\hline Non-inflammatory (OA) & $10.0(2)$ & $6.2(6)$ & \\
\hline FM without CTD & $0(0)$ & $9.3(9)$ & \\
\hline Hydroxychloroquine & $65.0(13)$ & $49.5(48)$ & \\
\hline Methotrexate & $15.0(3)$ & $7.2(7)$ & \\
\hline Mycophenolate & 0.0 & 0.0 & \\
\hline Azathioprine & $5.0(1)$ & $5.2(5)$ & \\
\hline Other DMARDs (leflunomide and sulfasalazine) & $5.0(1)$ & $4.1(4)$ & \\
\hline Belimumab & $5.0(1)$ & $4.1(4)$ & \\
\hline RA biologic & $5.0(1)$ & $16.5(16)$ & \\
\hline \multicolumn{4}{|l|}{ Medications at $\mathrm{t}=2(\%)$} \\
\hline Belimumab & $0(0)$ & $4.1(4)$ & ns \\
\hline Change in diagnosis at $\mathrm{t}=2$ & $80.0(16)$ & $28.9(28)$ & $<0.0001$ \\
\hline
\end{tabular}

CTD, connective tissue disease; DMARD, disease-modifying antirheumatic drug; FM, fibromyalgia ; ns, not significant; OA, osteoarthritis; RA, rheumatoid arthritis; UCTD, undifferentiated connective tissue disease.

in $16 / 20(80 \%)$ of patients with a positive test, compared with 28/97 (28.9\%) of patients with a non-positive test $(\mathrm{p}<0.0001)$ (table 1 and figure $2 \mathrm{~A})$. Furthermore, the diagnoses in $13 / 20(65 \%)$ of patients with a positive AVISE test changed to SLE, compared with 10/97 $(10.3 \%)$ of patients with a non-positive test $(\mathrm{p}<0.0001)$ (figure 2B). In comparison, the diagnosis to SLE changed in 3 out of 4 patients with a positive dsDNA IFA (75\%) at baseline vs $20 / 113(17.7 \%)$ without positive dsDNA IFA $(p=0.02)$. The two other most common diagnoses changes were to RA $(1 / 20$, or $5 \%$ of positive patients vs $8 / 97$, or $8.2 \%$ of non-positive patients, $\mathrm{p}=\mathrm{ns}$ ) and UCTD $(1 / 20$, or $5 \%$ of positive patients and $6 / 97$, or $6.1 \%$ of negative patients, $\mathrm{p}=\mathrm{ns}$ ) (data not shown). Of note, none of the nine patients who had a new RA diagnosis at 2 years had a positive CCP test at baseline.
Patients without an established baseline diagnosis who tested positive fulfilled significantly more total SLICC classification criteria at $\mathrm{t}=0(3.8 \pm 2.1)$ than those who tested non-positive $(1.9 \pm 1.1) \quad(\mathrm{p}=0.001) \quad$ (table 2$)$. Similarly, at 2 years, positive patients fulfilled a total of $4.5 \pm 2.2$ criteria, compared with $2.1 \pm 1.2$ in non-positive patients $(\mathrm{p}<0.0001)$ (table 2). Looking at the clinical classification criteria alone, scores were still significantly higher in the positive group at baseline $(2.1 \pm 1.5$ vs $1.1 \pm 0.8, \mathrm{p}=0.015)$ and at 2 years $(2.4 \pm 1.6$ vs $1.3 \pm 0.8, \mathrm{p}=0.006)$ (table 2$)$.

For comparison, we examined the sensitivity, specificity, positive predictive value (PPV) and negative predictive value (NPV) of traditional biomarkers for SLE (dsDNA, $\mathrm{C} 3$ and C4), ANA and AVISE at $\mathrm{t}=0$ in predicting a diagnosis of SLE at $\mathrm{t}=2$. Elevated dsDNA, low C3 and low C4 at $\mathrm{t}=0$ had good specificity $(>90 \%)$, but poor sensitivity 


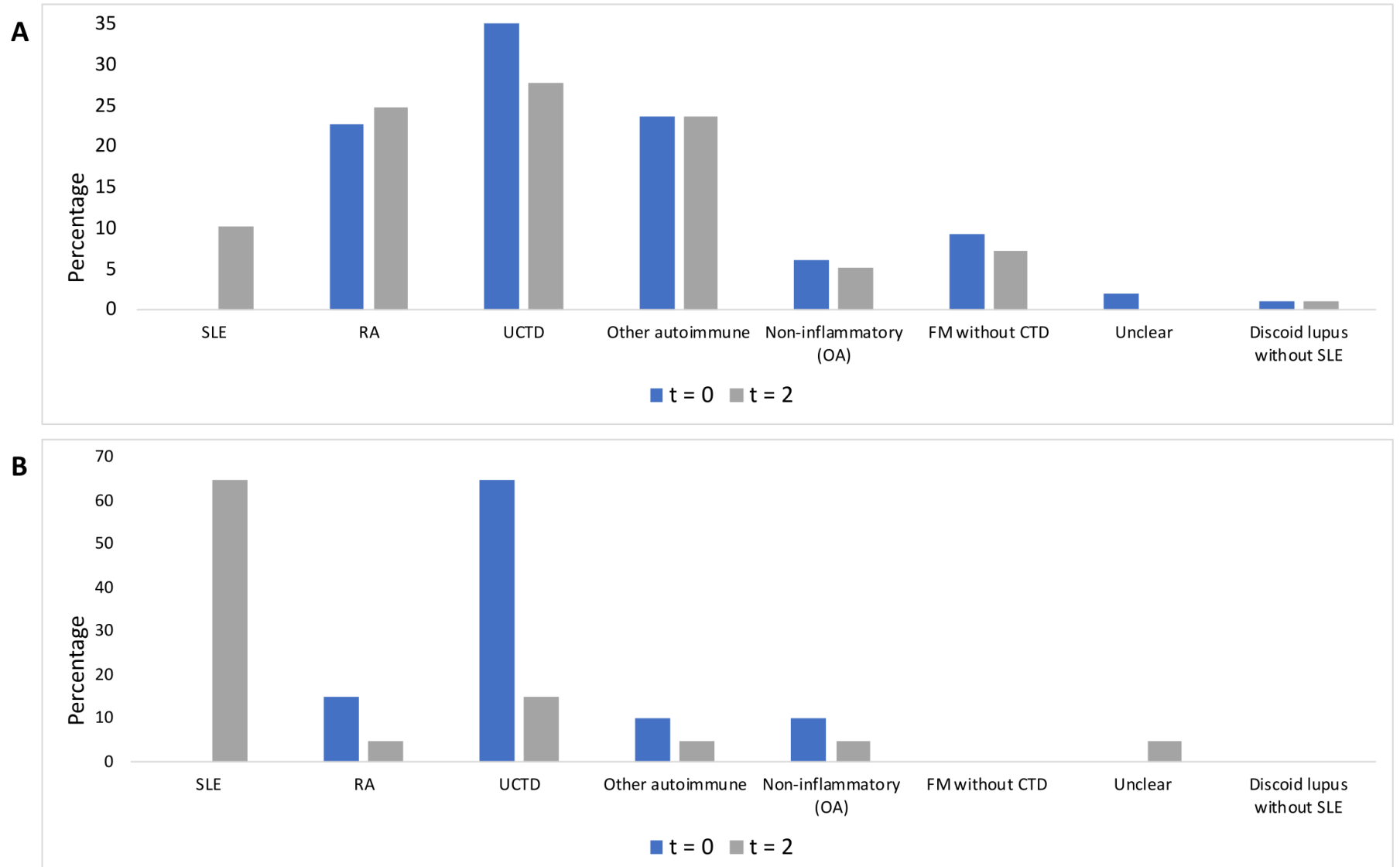

Figure 1 (A) Suspected diagnoses at $\mathrm{t}=0$ and $\mathrm{t}=2$ for AVISE-non-positive patients without an established diagnosis of SLE. (B) Suspected diagnoses at $\mathrm{t}=0$ and $\mathrm{t}=2$ for AVISE-positive patients without an established diagnosis of SLE. CTD, connective tissue disease; OA, osteoarthritis; RA, rheumatoid arthritis; UCTD, undifferentiated connective tissue disease.

$(<20 \%)$ for predicting a diagnosis of SLE at $\mathrm{t}=2$; in contrast, ANA positivity at $\mathrm{t}=0$ had good sensitivity $(87 \%)$ but low specificity $(39 \%$ ) (online supplementary table 1). AVISE positivity demonstrated a specificity of $93 \%$, with a sensitivity of $57 \%$, PPV of $65 \%$ and NPV of $90 \%$ (online supplementary table 1). Using logistic regression, only AVISE positivity was significantly associated with a confirmed diagnosis of SLE at $\mathrm{t}=2$, with an OR of 10.7 (95\% CI 2.6 to $44.9, \mathrm{p}=0.001$ ) (online supplementary table 2).

\section{Patients with a positive AVISE test are more likely to accumulate damage}

We also examined SDI at baseline and 2 years. In patients without an established SLE diagnosis at baseline, there were no significant differences in SDI at $\mathrm{t}=0$; however, at $\mathrm{t}=2$, positive patients had significantly greater damage $(1.9 \pm 1.3$ vs $1.03 \pm 1.3, p=0.01)$ (table 2$)$. When looking for any evidence of damage accumulation, at $\mathrm{t}=0,13 / 20$ $(65 \%)$ of positive patients had SDI $>0$, compared with
A

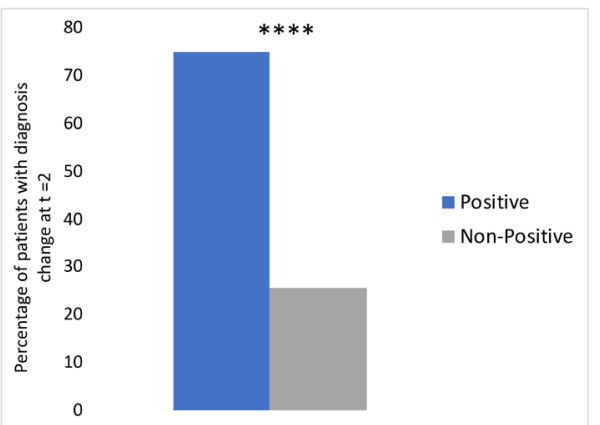

B

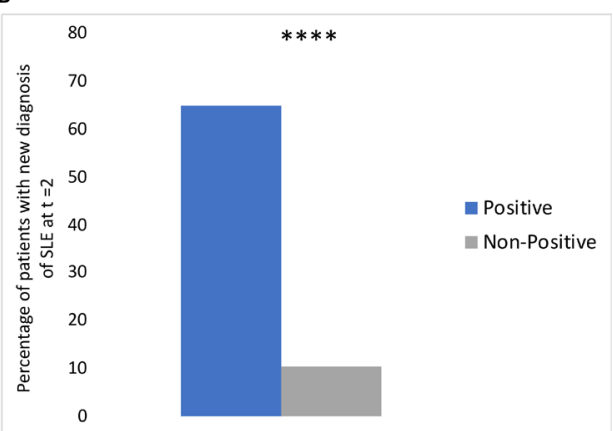

2 (A) Percentage of AVISE-positive and AVISE-non-positive patients without a diagnosis of SLE at baseline whose diagnosis changed at $t=2$. (B) Percentage of AVISE-positive and AVISE-non-positive patients without a diagnosis of SLE at baseline who developed SLE by $t=2{ }^{\star \star \star \star \star} p<0.0001$. 
Table 2 Number of ACR/SLICC classification criteria fulfilled and SDI at baseline and 2 years for AVISE positive and nonpositive patients

\begin{tabular}{|c|c|c|c|c|}
\hline & Time points & $\begin{array}{l}\text { Positive } \\
(n=20)\end{array}$ & $\begin{array}{l}\text { Non-positive } \\
(\mathrm{n}=97)\end{array}$ & $\begin{array}{l}\text { P value } \\
\text { (Student's } \\
\text { t-test) }\end{array}$ \\
\hline \multirow[t]{2}{*}{ SLICC/ACR criteria (total) } & $\mathrm{t}=0$ & $3.8 \pm 2.1$ & $1.9 \pm 1.1$ & 0.001 \\
\hline & $t=2$ & $4.5 \pm 2.2$ & $2.1 \pm 1.2$ & $<0.0001$ \\
\hline \multirow{2}{*}{$\begin{array}{l}\text { SLICC/ACR criteria } \\
\text { (clinical) }\end{array}$} & $\mathrm{t}=0$ & $2.1 \pm 1.5$ & $1.1 \pm 0.8$ & 0.015 \\
\hline & $\mathrm{t}=2$ & $2.4 \pm 1.6$ & $1.3 \pm 0.8$ & 0.006 \\
\hline $\begin{array}{l}\text { SDI (not including patients with established } \\
\text { SLE at baseline) }\end{array}$ & $t=2$ & $1.9 \pm 1.3$ & $1.03 \pm 1.3$ & 0.01 \\
\hline \multirow[t]{2}{*}{$\begin{array}{l}\text { SDI (including patients with established } \\
\text { SLE at baseline) }\end{array}$} & $t=0$ & $\begin{array}{l}(n=31) \\
1.8 \pm 2.02\end{array}$ & $\begin{array}{l}(n=107) \\
0.8 \pm 1.2\end{array}$ & 0.01 \\
\hline & $t=2$ & $2.2 \pm 2.0$ & $1.1 \pm 1.3$ & 0.006 \\
\hline
\end{tabular}

ACR, American College of Rheumatology; SDI, Systemic Lupus International Collaborating Clinics Damage Index; SLICC, Systemic Lupus International Collaborating Clinics.

$43(44.3 \%)$ of non-positive patients $(\mathrm{p}=0.09)$. At 2 years, $18 / 20(90.0 \%)$ of positive patients had SDI $>0$, compared with $54(55.7 \%)$ of non-positive patients $(\mathrm{p}=0.005)$.

When looking at the entire cohort, including patients with an established SLE at baseline, we found that SDI was significantly higher in patients with a positive AVISE test at both baseline $(\mathrm{p}=0.01)$ and 2 years $(0.006)$ (table 2$)$.

There were no significant differences in medication regimens between positive and non-positive patients at either $\mathrm{t}=0$ or $\mathrm{t}=2$, with the exception of more frequent MMF use in AVISE positive patients at $\mathrm{t}=2$ (table 1 ).

\section{Correlation of complement levels and AVISE biomarkers with clinical outcomes}

At $\mathrm{t}=0$, BC4d and EC4d levels significantly correlated with the total number of SLICC criteria $(r=0.33$, $\mathrm{p}<0.0001$, and $\mathrm{r}=0.23, \mathrm{p}=0.008$, respectively). These correlations remained significant when examining the number of clinical SLICC criteria $(\mathrm{r}=0.28, \mathrm{p}=0.001$ for BC4d and $\mathrm{r}=0.17, \mathrm{p}=0.04$ for EC4d). At $\mathrm{t}=2$, BC4d and EC4d remained correlated with the total SLICC and clinical SLICC criteria $(\mathrm{p}<0.02$ for all $)$.

C3 and C4 levels and titers of dsDNA antibodies at $\mathrm{t}=0$ correlated with the number of total SLICC criteria

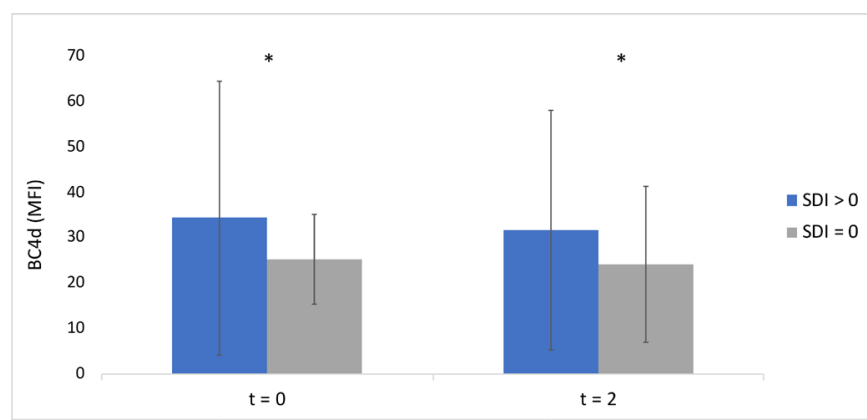

Figure $3 \quad \mathrm{BC} 4 d$ levels in patients with damage (SDI $>0)$ and those without damage $(\mathrm{SDI}=0)$ at $\mathrm{t}=0$ and $\mathrm{t}=2$. $\mathrm{BC} 4 \mathrm{~d}$, B-cell bound C4d; MFI, mean fluorescence intensity; SDI, Systemic Lupus International Collaborating Clinics Damage Index. $(\mathrm{r}=-0.33, \mathrm{p}=0.004 ; \mathrm{r}=-0.38, \mathrm{p}=0.001 ;$ and $\mathrm{r}=0.34, \mathrm{p}<0.0001$, respectively) and clinical SLICC criteria $(\mathrm{r}=-0.22$, $\mathrm{p}=0.05 ; \mathrm{r}=-0.26, \mathrm{p}=0.03$; and $\mathrm{r}=0.19, \mathrm{p}=0.03$, respectively) at $\mathrm{t}=0$. All four biomarkers at $\mathrm{t}=0$ also correlated with the total and SLICC criteria at $\mathrm{t}=2(\mathrm{p}<0.05)$.

BC4d levels correlated with the total SDI at $\mathrm{t}=0 \quad(\mathrm{r}=0.25$, $\mathrm{p}=0.003)$ and $\mathrm{t}=2(\mathrm{r}=0.26, \mathrm{p}=0.002) ; \mathrm{C} 4$ levels also inversely correlated with SDI at $\mathrm{t}=0(-0.26, \mathrm{p}=0.03)$ and $\mathrm{t}=2(-0.25$, $\mathrm{p}=0.03)$. EC4d, C3, and dsDNA levels did not significantly correlate with SDI at $\mathrm{t}=0$ or $\mathrm{t}=2$.

At $\mathrm{t}=0$, patients who had experienced damage (SDI $>0$ ) had significantly higher BC4d levels (mean fluorescence intensity (MFI) 34.7 \pm 30.1 ) than those without damage (MFI 24.1 \pm 20.4$) \quad(\mathrm{p}=0.03) \quad$ (figure 3). At $\mathrm{t}=2$, patients who had experienced damage (SDI $>0$ ) had significantly higher baseline BC4d levels (MFI 32.0 \pm 26.5 ) than those without damage (MFI 24.0 \pm 17.2$) \quad(\mathrm{p}=0.04)$ (figure 3). There were no significant differences in EC4d, C3, C4 or dsDNA levels among patients with and without damage at $\mathrm{t}=0$ or $\mathrm{t}=2$ (data not shown).

\section{DISCUSSION}

The AVISE CTD test has been demonstrated to aid in differentiation of SLE diagnosis from other CTDs and FM. ${ }^{15} 16$ We present here the first study to evaluate its clinical utility in a real-world cohort. Patients with a positive AVISE test who did not have a confirmed diagnosis at baseline were more likely to develop a firm SLE diagnosis within 2 years after testing. The AVISE test demonstrated high specificity and good sensitivity for predicting the development of SLE at 2 years and was the only biomarker in multivariate analysis to be associated with a diagnosis of SLE at 2 years. Interestingly, positive patients were also more likely to develop damage at baseline and 2 years. Furthermore, patients with damage had significantly greater levels of BC4d at both baseline and 2 years than those without. Taken together, these results suggest 
that AVISE positivity is predictive of SLE diagnosis and damage progression.

Our study represents an advance in research regarding the clinical utility of the AVISE CTD test. While previous studies have shown that the test improves the sensitivity and specificity of diagnosing SLE in cases versus controls ${ }^{15}{ }^{16}$ we have demonstrated that AVISE positivity can not only predict development of SLE within a 2-year interval, but also with equal or greater specificity and greater sensitivity than traditional biomarkers. AVISE positivity at baseline in this cohort also demonstrated a 10.7-fold increased OR for a confirmed SLE diagnosis at 2 years. Furthermore, we have shown that AVISE positivity may be used to predict damage progression as measured by SDI, which has not been previously investigated. This ability distinguishes the AVISE test from other serological autoantibody tests of SLE, such as the SLE Key Rule-Out test, which distinguishes healthy subjects from SLE patients with high sensitivity and negative predictive value but has not been shown to correlate with disease activity or damage progression. ${ }^{17} 18$ Another recent prospective study of the clinical utility of the AVISE test demonstrated that AVISE results influenced diagnostic and treatment impressions, as patients who tested positive were more likely to be started on hydroxychloroquine or prednisone, while negative test results strengthened physician confidence that a diagnosis of SLE was unlikely. ${ }^{19}$ In our study, both positive and negative patients continued to be monitored clinically; we noted that while patients who tested positive were more likely to go on to fulfil diagnostic criteria for SLE and accumulate damage, several patients who initially tested negative went on to develop SLE. Thus, while AVISE testing can improve clinical decision making, it is still important to interpret results in the context of a patient's clinical presentation.

In contrast, C3 and C4 levels did not consistently correlate with SDI damage progression in our cohort. These findings corroborate previous studies that identified the limitations of relying on hypocomplementemia as a measure of disease damage progression in SLE. ${ }^{10} 11$ This is similar to other published studies. ${ }^{20}{ }^{21}$ Instead, our study strengthens the potential of CB-CAPs in the diagnosis and monitoring of SLE. Prior studies have shown that elevated CB-CAP levels increase the sensitivity and specificity of diagnosing SLE and predict higher levels of disease activity as measured by the Safety of Estrogens in Lupus National Assessment-Systemic Lupus Erythematosus Disease Activity Index (SELENA-SLEDAI) score. ${ }^{1012} 14$ In our study, we used SDI to evaluate disease damage progression. By accounting for sequelae from SLE itself as well as medications, this measure permits a more holistic evaluation of disease consequences. By analysing BC4d and EC4d levels separately, we showed that BC4d levels are the primary predictor of disease progression. This finding reinforces the clinical utility of using CB-CAPs levels, particularly BC4d, in monitoring disease progression.
The strengths of our study include the use of patients from a single rheumatology provider, which controlled for heterogeneity in diagnosis and management. The study was also performed independently of the manufacturers of the AVISE testing, avoiding any bias in study design, data collection and analysis. The cohort consisted of patients from a university-affiliated community rheumatology clinic, strengthening the real-world applications of our results.

The primary limitation of our study is the relatively small sample size, which was due to the recent introduction of AVISE testing into rheumatology practice and the observational nature of the study. Nonetheless, our study was still able to detect significant differences and correlations at the $\mathrm{p}<0.0001$ level. In addition, due to the retrospective nature of this study, we were unable to exclude bias in the selection of therapeutic regimens in positive and non-positive patients; differences in treatment may have affected the probability of a changed diagnosis and damage score. However, only MMF use was significantly different at $\mathrm{t}=2$ in AVISE positive patients, suggesting that more aggressive use of corticosteroids or other medications in the positive group was not a major contributor to clinical outcomes. Another limitation of our study was our use of the SDI to evaluate damage in non-SLE patients. We used it as a universal marker of damage in our study because the AVISE test was designed specifically for SLE. Although SDI has not been validated for other rheumatological diagnoses, we still found significant differences in damage progression between positive and non-positive patients.

Future studies will evaluate the utility of AVISE in predicting longitudinal disease activity and flares. These results will determine whether AVISE positivity can predict disease activity at shorter intervals. In addition, a larger patient cohort would serve as a validation dataset to confirm our findings, allow generalisation to a broader patient population and permit analysis of AVISE results with other sequelae of SLE, such as neurological or cardiovascular events. Lastly, longer term follow-up of this patient cohort may reveal further associations between AVISE positivity and SLE development or progression.

Contributors EL, MT and MMcM designed the study. EL and MMcM analysed the results and wrote the manuscript.

Funding This work was funded by a David Geffen School of Medicine Research Grant. Note that Exagen diagnostics did not provide funding for the study and did not have any input into study design, study procedures, data analysis or interpretation of results.

Competing interests None declared.

Patient consent for publication Not required.

Ethics approval Ethical approval for this retrospective chart review was obtained from the UCLA Institutional Review Board (protocol number 17-001607).

Provenance and peer review Not commissioned; externally peer reviewed. Data availability statement Data are available upon reasonable request.

Open access This is an open access article distributed in accordance with the Creative Commons Attribution Non Commercial (CC BY-NC 4.0) license, which permits others to distribute, remix, adapt, build upon this work non-commercially, and license their derivative works on different terms, provided the original work is 
properly cited, appropriate credit is given, any changes made indicated, and the use is non-commercial. See: http://creativecommons.org/licenses/by-nc/4.0/.

\section{ORCID iD}

Maureen McMahon http://orcid.org/0000-0002-2910-7640

\section{REFERENCES}

1 Tsokos GC. Systemic lupus erythematosus. N Engl J Med 2011;365:2110-21.

2 Petri M, Orbai A-M, Alarcón GS, et al. Derivation and validation of the systemic lupus international collaborating clinics classification criteria for systemic lupus erythematosus. Arthritis Rheum 2012;64:2677-86.

3 Mosca M, Tani C, Vagnani S, et al. The diagnosis and classification of undifferentiated connective tissue diseases. J Autoimmun 2014;4849:50-2.

4 Oglesby A, Korves C, Laliberté F, et al. Impact of early versus late systemic lupus erythematosus diagnosis on clinical and economic outcomes. Appl Health Econ Health Policy 2014;12:179-90.

5 Romero-Diaz J, Isenberg D, Ramsey-Goldman R. Measures of adult systemic lupus erythematosus: updated version of British Isles lupus assessment group (BILAG 2004), European consensus lupus activity measurements (ECLAM), systemic lupus activity measure, revised (SLAM-R), systemic lupus activity Questi. Arthritis Care Res 2011;63:S37-46

6 Fernando MMA, Isenberg DA. How to monitor SLE in routine clinical practice. Ann Rheum Dis 2005;64:524-7.

7 Gladman D, Ginzler E, Goldsmith C, et al. The development and initial validation of the systemic lupus international collaborating Clinics/American College of rheumatology damage index for systemic lupus erythematosus. Arthritis Rheum 1996;39:363-9.

8 Swaak AJ, Groenwold J, Aarden LA, et al. Prognostic value of antidsDNA in SLE. Ann Rheum Dis 1982;41:388-95 http://www.ncbi.nlm. nih.gov/pubmed/6981385

9 Steiman AJ, Gladman DD, Ibañez D, et al. Prolonged serologically active clinically quiescent systemic lupus erythematosus: frequency and outcome. J Rheumatol 2010;37:1822-7.
10 Putterman C, Furie R, Ramsey-Goldman R, et al. Cell-Bound complement activation products in systemic lupus erythematosus: comparison with anti-double-stranded DNA and standard complement measurements. Lupus Sci Med 2014;1:e000056.

11 Walport MJ. Complement and systemic lupus erythematosus. Arthritis Res 2002;4 Suppl 3:S279.

12 Liu C-C, Manzi S, Kao AH, et al. Cell-Bound complement biomarkers for systemic lupus erythematosus: from benchtop to bedside. Rheum Dis Clin North Am 2010;36:161-72.

13 Hui-Yuen JS, Gartshteyn Y, Ma M, et al. Cell-Bound complement activation products (CB-CAPs) have high sensitivity and specificity in pediatric-onset systemic lupus erythematosus and correlate with disease activity. Lupus 2018;27:2262-8.

$14 \mathrm{Kao} \mathrm{AH}$, Navratil JS, Ruffing MJ, et al. Erythrocyte C3d and C4d for monitoring disease activity in systemic lupus erythematosus. Arthritis Rheum 2010;62:837-44.

15 Mossell J, Goldman JA, Barken D, et al. The Avise lupus test and cell-bound complement activation products aid the diagnosis of systemic lupus erythematosus. Open Rheumatol J 2016;10:71-80.

16 Wallace DJ, Silverman SL, Conklin J, et al. Systemic lupus erythematosus and primary fibromyalgia can be distinguished by testing for cell-bound complement activation products. Lupus Sci Med 2016;3:e000127.

17 Putterman C, Wu A, Reiner-Benaim A, et al. SLE-key(®) ruleout serologic test for excluding the diagnosis of systemic lupus erythematosus: Developing the ImmunArray iCHIP(®). J Immunol Methods 2016;429:1-6.

18 Putterman C, Pisetsky DS, Petri M, et al. The SLE-key test serological signature: new insights into the course of lupus. Rheumatology 2018;57:1632-40.

19 Wallace DJ, Alexander RV, O'Malley T, et al. Randomised prospective trial to assess the clinical utility of multianalyte assay panel with complement activation products for the diagnosis of SLE. Lupus Sci Med 2019;6:e000349.

20 Raymond W, Eilertsen G, Nossent J. Hypocomplementemia as a risk factor for organ damage Accrual in patients with systemic lupus erythematosus. J Immunol Res 2018;2018:1-7.

21 Gandino IJ, Scolnik M, Bertiller E, et al. Complement levels and risk of organ involvement in patients with systemic lupus erythematosus. Lupus Sci Med 2017;4:e000209. 